\title{
Medium amplitude limit cycles of some classes of generalized Liénard systems
}

\author{
Salomón Rebollo-Perdomo \\ Centre de Recerca Matemàtica, \\ 08193 Bellaterra, Barcelona, Spain. \\ srebollo@crm.cat
}

\section{Introduction and statement of the results}

The bifurcation of limit cycles by perturbing a planar system which has a continuous family of cycles, i.e. periodic orbits, has been an intensively studied phenomenon; see for instance $[13,16,2]$ and references therein. The simplest planar system having a continuous family of cycles is the linear center, and a special family of its perturbations is given by the generalized polynomial Liénard systems:

$$
\dot{x}=y+\sum_{i=1}^{\mu} \varepsilon^{i} F_{i}(x), \quad \dot{y}=-x+\sum_{i=1}^{\nu} \varepsilon^{i} g_{i}(x),
$$

where $\mu, \nu \in \mathbb{N}, g_{i}(x)$ and $F_{i}(x)$ are polynomials for $i \geq 1$, and $\varepsilon$ is a small parameter.

The classical and generalized Liénard systems appear very often in several branches of science and engineering, as biology, chemistry, mechanics, electronics, etc., see for instance [20] and references therein. In particular Liénard systems are frequent specially in physiological processes, see for instance [10]. Further, some planar systems can be transformed into (generalized) Liénard systems, see for example [5, 15]. In addition, the generalized polynomial Liénard systems is one of the most considered families in the study of limit cycles, see [18].

We assume $F_{\mu}(x) \not \equiv 0$ and $g_{\nu}(x) \not \equiv 0$, then we define

$$
m=\max _{1 \leq i \leq \mu}\left\{\operatorname{deg} F_{i}(x)\right\}
$$

and

$$
n=\max _{1 \leq i \leq \nu}\left\{\operatorname{deg} g_{i}(x)\right\} .
$$

For a small enough $\varepsilon$, let $\mathcal{H}_{\nu}^{\mu}(m, n)$ be the maximum number of limit cycles of $\left(1_{\varepsilon}\right)$ that bifurcate from cycles of the linear center $\left(1_{0}\right)$, i.e. the maximum number of medium amplitude limit cycles which can bifurcate from $\left(1_{0}\right)$ under the perturbation $\left(1_{\varepsilon}\right)$, in short

$$
\mathcal{H}_{\nu}^{\mu}(m, n)=\left\{\begin{array}{l}
\text { Maximum number of medium } \\
\text { amplitude limit cycles of }\left(1_{\varepsilon}\right)
\end{array}\right\}
$$

The main problem concerning $\mathcal{H}_{\nu}^{\mu}(m, n)$ is finding its exact value. 
We are interested in $\nu \geq 1$ because when $g_{i}(x) \equiv 0$ for all $i \geq 1$ the maximum number of medium amplitude limit cycles of $\left(\overline{1}_{\varepsilon}\right)$ is well-known. Indeed, if we denote by $\mathcal{H}_{0}^{\mu}(m)$ the maximum number of medium amplitude limit cycles of $\left(1_{\varepsilon}\right)$ in such a case, then we know from $[17]$ that $\mathcal{H}_{0}^{1}(m) \geq[(m-1) / 2]$, where [.] denotes the integer part function. Moreover, by following [7, Theorem 3.1] we can prove that $\mathcal{H}_{0}^{\mu}(m)=[(m-1) / 2]$ for $\mu \geq 1$. An explicit proof of this statement is provided in [1, Section 3.2.2] because it is known that the cyclicity of a non-degenerated center (as in our case) coincide with the cyclicity of the open period annulus surrounding it. See for instance [9]. Theorem 1 (below) is a generalization of this result, and it also improves the results of Section 4.3.2 in [1] which prove that $\mathcal{H}_{\nu}^{\mu}(m, n)=[(m-1) / 2]$ for some families of generalized Liénard systems. A rewiev about the results conserning small and medium amplitude limit cycles of $\left(1_{\varepsilon}\right)$ is given in [19], where is also proved that

$$
\begin{aligned}
& \mathcal{H}_{1}^{1}(m, n) \geq\left[\frac{m-1}{2}\right], \\
& \mathcal{H}_{2}^{2}(m, n) \geq \max \left\{\left[\frac{m-1}{2}\right],\left[\frac{m}{2}\right]+\left[\frac{n}{2}\right]-1\right\}, \\
& \mathcal{H}_{3}^{3}(m, n) \geq\left[\frac{m+n-1}{2}\right] .
\end{aligned}
$$

However, the exact values of $\mathcal{H}_{1}^{1}(m, n), \mathcal{H}_{2}^{2}(m, n)$, and $\mathcal{H}_{3}^{3}(m, n)$ were not reported there.

In this paper we give the exact value of $\mathcal{H}_{\nu}^{\mu}(m, n)$ for two subfamilies of $\left(1_{\varepsilon}\right)$. More precisely, we consider the families:

$$
\mathcal{G L} 1:=\left\{\begin{array}{c}
\text { Systems }\left(1_{\varepsilon}\right) \text { assuming that } \\
g_{i}(x) \text { is odd for } 1 \leq i \leq \nu
\end{array}\right\}
$$

and

$$
\mathcal{G L} 2:=\left\{\begin{array}{l}
\text { Systems }\left(1_{\varepsilon}\right) \text { assuming that } \\
F_{i}(x) \text { is even for } \mu_{0}<i \leq \mu
\end{array}\right\},
$$

where $\mu_{0}$ is the smallest integer with $1 \leq \mu_{0} \leq \mu$ such that $F_{\mu_{0}}(x) \not \equiv 0$.

We will give the exact values of $\tilde{\mathcal{H}}_{\nu}^{\mu}(m, n)$ and $\overline{\mathcal{H}}_{\nu}^{\mu}(m, n)$ the maximum number of medium amplitude limit cycles of systems in $\mathcal{G} \mathcal{L} 1$ and $\mathcal{G} \mathcal{L} 2$, respectively. We note that if $\mu_{0}=\mu$, then $\overline{\mathcal{H}}_{\nu}^{\mu}(m, n)=\mathcal{H}_{\nu}^{\mu}(m, n)$.

Our main result is the following.

Theorem 1. The following statements hold.

(a) The exact value of $\tilde{\mathcal{H}}_{\nu}^{\mu}(m, n)$ is $\left[\frac{m-1}{2}\right]$. Moreover, for each $s$ with $0 \leq s \leq\left[\frac{m-1}{2}\right]$ there exist systems in $\mathrm{GL1}$ having exactly s hyperbolic limit cycles.

(b) The exact value of $\overline{\mathcal{H}}_{\nu}^{\mu}(m, n)$ is either $\left[\frac{m-1}{2}\right]$ if $m$ is odd or $\left[\frac{m}{2}\right]+\left[\frac{n}{2}\right]-1$ if $m$ is even. Moreover, for each $s$ with $0 \leq s \leq\left[\frac{m}{2}\right]+\left[\frac{n}{2}\right]-1$ there exist systems in $\mathcal{G L} 2$ having exactly s hyperbolic limit cycles.

The assumptions on $g_{i}(x)$ and $F_{i}(x)$ in definitions of $\mathcal{G L} 1$ and $\mathcal{G L} 2$, respectively, are necessary. Otherwise, we can construct systems $\left(1_{\varepsilon}\right)$ having more medium amplitude limit cycles, see Remark 1 in Section 3.

Theorem 1 is a generalization of Theorem 1.1 in [22], where the case $\mu=\nu=1$ was considered. We note that in such a case $\overline{\mathcal{H}}_{1}^{1}(m, n)=\mathcal{H}_{1}^{1}(m, n)$. Hence Theorem 1.(b) gives the exact value of $\mathcal{H}_{1}^{1}(m, n)$.

The proof of Theorem 1 is based on computing the maximum number of isolated zeros of the first non-vanishing Poincaré-Pontryagin-Melnikov function of the displacement function of $\left(1_{\varepsilon}\right)$, by taking into account the restrictions: $g_{i}(x)$ odd for $1 \leq i \leq \nu$ and $F_{i}(x)$ even for $\mu_{0}<i \leq \mu$, respectively. 
The paper is organized as follows. In Section 2 we recall the definition of the displacement function of $\left(1_{\varepsilon}\right)$, as well as the algorithm to compute the Poincaré-Pontryagin-Melnikov functions. Preliminary results that allow us to provide elementary proofs of the main result are given in Section 3. Finally, in Section 4 we will prove Theorem 1.

\section{Poincaré-Pontryagin-Melnikov functions}

The linear center $\left(1_{0}\right)$ is the Hamiltonian system associated to the polynomial $H=\left(x^{2}+y^{2}\right) / 2$; hence its cycles are the circles $\gamma_{c}=\{H-c=0\}$ with $c>0$. By using $c$ as a parameter, the first return map of $\left(1_{\varepsilon}\right)$ can be expressed in terms of $\varepsilon$ and $c: \mathcal{P}(\varepsilon, c)$. Therefore the corresponding displacement function $L(\varepsilon, c)=\mathcal{P}(\varepsilon, c)-c$ is analytic for small enough $\varepsilon$ and can be written as the power series in $\varepsilon$

$$
L(\varepsilon, c)=\varepsilon L_{1}(c)+\varepsilon^{2} L_{2}(c)+O\left(\varepsilon^{3}\right),
$$

where $L_{i}(c)$ with $i \geq 1$ is the Poincaré-Pontryagin-Melnikov function of order $i$, which is defined for $c \geq 0$.

Let $L_{k}(c)$ with $k \geq 1$ be the first non-vanishing coefficient in (2). The zeros of $L_{k}(c)$ are important in the study of medium amplitude limit cycles of $\left(1_{\varepsilon}\right)$ because of the Poincaré-Pontryagin-Andronov criterion: The maximum number of isolated zeros, counting multiplicities, of $L_{k}(c)$ is an upper bound for $\mathcal{H}_{\nu}^{\mu}(m, n)$. Furthermore each simple zero $c_{0}$ of $L_{k}(c)$ corresponds to one and only one limit cycle of $\left(1_{\varepsilon}\right)$ with $\varepsilon$ small enough bifurcating from the cycle $\gamma_{c_{0}}$.

Now, we will recall the algorithm to compute the functions $L_{i}(c)$. System $\left(1_{\varepsilon}\right)$ can be written as

$$
\dot{x}=y, \quad \dot{y}=-x+\sum_{i \geq 1} \varepsilon^{i}\left(g_{i}(x)+f_{i}(x) y\right)
$$

where $f_{i}(x)=F_{i}^{\prime}(x)$, or equivalently as

$$
d H-\varepsilon \omega_{1}-\varepsilon^{2} \omega_{2}-\cdots=0
$$

with $\omega_{i}=\left(g_{i}(x)+f_{i}(x) y\right) d x$ and $\omega_{i} \equiv 0$ for $i>\max \{\mu, \nu\}$.

As we know, $L_{1}(c)$ is given by the classical Poincaré-Pontryagin formula $L_{1}(c)=\int_{\gamma_{c}} \omega_{1}$. A construction to compute the second order Poincaré-Pontryagin-Melnikov function of a perturbed system of the form $d H-\varepsilon \omega_{1}$ with $\omega_{1}$ an arbitrary polynomial 1-form was given by Yakovenko [1995]. After, Françoise [1996] gave the algorithm to know the Poincaré-Pontryagin-Melnikov function of any order of $d H-\varepsilon \omega_{1}$. Finally, Iliev [1999] gave the result for computing the higher order Poincaré-Pontryagin-Melnikov functions of a perturbed system of the form $d H-\varepsilon \omega_{1}-\varepsilon^{2} \omega_{2}-\cdots=0$, where $\omega_{i}$ for $i \geq 1$ are arbitrary polynomial 1 -forms. His result is the following.

Theorem 2. [11]. If $k \geq 2$ and $L_{1}(c) \equiv \cdots \equiv L_{k-1}(c) \equiv 0$, then there are polynomials $q_{1}, \ldots, q_{k-1}$ and $Q_{1}, \ldots, Q_{k-1}$ such that $\Omega_{1}=d Q_{1}+q_{1} d H, \ldots, \Omega_{k-1}=d Q_{k-1}+q_{k-1} d H$, and

$$
L_{k}(c)=\int_{\gamma_{c}} \Omega_{k}
$$

where

$$
\Omega_{1}=\omega_{1}, \Omega_{l}=\omega_{l}+\sum_{i+j=l} q_{i} \omega_{j} \text {, and } 2 \leq l \leq k .
$$


The proof of this result easily follows from the Poincaré-Pontryagin formula, and the Ilyashenko-Gavrilov theorem ([12], [8]): If $\int_{\gamma_{c}} \omega=0$ for all $c \geq 0$, then $\omega=d Q+q d H$, where $Q$ and $q$ are polynomials, and by applying an induction argument. For a detailed proof, see for instance [11], [14].

On the other hand, we know from $[11]$ that $L_{k}(c)$ has at $\operatorname{most}[k(\max \{n, m\}-1) / 2]$ positive zeros, counting multiplicities. However, this result does not give the value of $\mathcal{H}_{\nu}^{\mu}(m, n)$ because the upper bound for $k$ depending on $\mu, \nu, m$, and $n$ is unknown. In addition, the number of isolated zeros of the first nonvanishing Poincaré-Pontryagin-Melnikov function does not provide the number of limit cycles of $\left(1_{\varepsilon}\right)$ with $\varepsilon$ small enough as shows next example.

Example 1. Consider the Liénard system

$$
\dot{x}=y+\varepsilon x-\varepsilon^{2} x^{3}, \quad \dot{y}=-x,
$$

or equivalently $d H-\varepsilon \omega_{1}-\varepsilon^{2} \omega_{2}=0 \quad$ with $\omega_{1}=y d x$ and $\omega_{2}=3 x^{2} y d x$, where $\varepsilon$ is a small parameter.

A simple computation gives $L_{1}(c)=\int_{\gamma_{c}} \omega_{1}=-2 \pi c$. Hence system $\left(5_{\varepsilon}\right)$ does not have limit cycles bifurcating from the cycles of the linear center. However, for any $\varepsilon>0$ small enough the system $\left(5_{\varepsilon}\right)$ has a limit cycle bifurcating from the infinity; more precisely, if we consider $\left(5_{\varepsilon}\right)$ on the Poincaré sphere $\mathbb{S}^{2}$, then the limit cycle bifurcates from the equator of $\mathbb{S}^{2}$ which is known as "the circle at infinity" or "points at infinity" of $\mathbb{R}^{2}$ [21]. Indeed, by using the function

$$
V_{\varepsilon}(x, y)=4 y^{2}+4 \varepsilon x\left(1-\varepsilon x^{2}\right) y+4 x^{2}-\frac{3}{\varepsilon^{2}}
$$

it is not difficult to prove that the $1 / V_{\varepsilon}(x, y)$ is a Dulac function for $\left(5_{\varepsilon}\right)$ in $\mathbb{R}^{2} \backslash\left\{V_{\varepsilon}(x, y)=0\right\}$; moreover, it is easy to see that for $\varepsilon \in(0,1)$ the curve $\left\{V_{\varepsilon}(x, y)=0\right\}$ has an oval surrounding the origin (the unique singularity of $\left.\left(5_{\varepsilon}\right)\right)$. Hence, $\mathbb{R}^{2} \backslash\left\{V_{\varepsilon}(x, y)=0\right\}$ has a 1-connected component $\widetilde{U}_{\varepsilon}$, then the generalized Bendixon-Dulac theorem [6] ensures that $\left(5_{\varepsilon}\right)$ has a hyperbolic limit cycle $\Gamma_{\varepsilon}$ in $\widetilde{U}_{\varepsilon}$ for each $\varepsilon \in(0,1)$. Thus, $\Gamma_{\varepsilon}$ contains the oval of $\left\{V_{\varepsilon}(x, y)=0\right\}$. See Section 4 of [4] for more details. Finally, a straightforward computation shows that the circle $x^{2}+y^{2}=1 /(2 \varepsilon)^{2}$ is contained in the bounded region limited by the oval of $\left\{V_{\varepsilon}(x, y)=0\right\}$. This implies that $\Gamma_{\varepsilon}$ bifurcates from the "the circle at infinity" of $\mathbb{R}^{2}$.

In next section we will give some properties on $\omega_{i}$ which will allow us to simplify the computation of the Poincaré-Pontryagin-Melnikov functions

\section{Preliminary results}

For computing $L_{k}(c)$ for $\left(1_{\varepsilon}\right)$ we will use the following two elementary lemmas whose proof is omitted.

Lemma 3. Let $P$ be a polynomial in the ring $\mathbb{R}\left[x^{2}, H\right]$. We define $\operatorname{deg}_{2} P$ to be the degree of $P$ in $\mathbb{R}\left[x^{2}, H\right]$.

(a) For $i, j \geq 0$ there are homogeneous polynomials $Q_{i j}, q_{i j} \in \mathbb{R}\left[x^{2}, H\right]$ with $\operatorname{deg}_{2} Q_{i j}=i+j$ and $\operatorname{deg}_{2} q_{i j}=$ $i+j-1$, such that

$$
H^{i} x^{2 j} d x=d\left(x Q_{i j}\right)+\left(x q_{i j}\right) d H
$$

or

$$
H^{i} x^{2 j+1} d x=d\left(x^{2} Q_{i j}\right)+\left(x^{2} q_{i j}\right) d H .
$$

If $i=0$, then $q_{i j} \equiv 0$. 
(b) For $i, j \geq 0$ there are homogeneous polynomials $Q_{i j}, q_{i j} \in \mathbb{R}\left[x^{2}, H\right]$ with $\operatorname{deg}_{2} Q_{i j}=i+j+1$ and $\operatorname{deg}_{2} q_{i j}=i+j$, such that

$$
H^{i} x^{2 j+1} y d x=d\left(y Q_{i j}\right)+\left(y q_{i j}\right) d H .
$$

(c) For $i, j \geq 0$ we have

$$
\int_{\gamma_{c}} H^{i} x^{2 j} y d x=\frac{-\pi c}{2^{j}(2 j+1)}\left(\begin{array}{c}
2(j+1) \\
j+1
\end{array}\right) c^{i+j} .
$$

Lemma 4. If $\omega, \eta \in \mathcal{A}$ and $q \in \mathcal{S}$ where

$$
\mathcal{A}:=\left\{(x A+x y B) d x \mid A, B \in \mathbb{R}\left[x^{2}, H\right]\right\}
$$

and

$$
\mathcal{S}:=\left\{x^{2} q_{1}+y q_{2} \mid q_{1}, q_{2} \in \mathbb{R}\left[x^{2}, H\right]\right\},
$$

then $\omega+\eta \in \mathcal{A}$ and $q \omega \in \mathcal{A}$.

The next two results are straightforward consequences of these two previous lemmas.

Corollary 5. If $\omega \in \mathcal{A}$, then $\int_{\gamma_{c}} \omega \equiv 0, \omega=d Q+q d H$ with $q \in \mathcal{S}$, and $q \omega \in \mathcal{A}$.

Corollary 6. If $P\left(x^{2}\right)=\sum_{r=0}^{d} p_{r} x^{2 r} \in \mathbb{R}\left[x^{2}\right]$, then

$$
\int_{\gamma_{c}} P\left(x^{2}\right) y d x=-\pi c \sum_{r=0}^{d}\left(\begin{array}{c}
2(r+1) \\
r+1
\end{array}\right) \frac{p_{r}}{2^{r}(2 r+1)} c^{r} .
$$

We now will prove two lemmas which will be useful in the proof of Theorem 1.

Lemma 7. Suppose $k \geq 2$. Then the following statements hold.

(a) $\omega_{l} \in \mathcal{A}$ for $1 \leq l \leq k-1$ if and only if $\Omega_{l} \in \mathcal{A}$ for $1 \leq l \leq k-1$, where $\Omega_{l}$ is defined as in (4).

(b) If $\Omega_{l} \in \mathcal{A}$ for $1 \leq l \leq k-1$, then $L_{1}(c) \equiv \cdots \equiv L_{k-1}(c) \equiv 0$ and $L_{k}(c)=\int_{\gamma_{c}} \omega_{k}$.

Proof. (a) We proceed by induction on $k$. If $k=2$, then statement $(a)$ is true because $\Omega_{1}=\omega_{1} \in \mathcal{A}$. We now assume that the statement is true for $k-1$, and we will prove it for $k$.

From the induction hypothesis it follows that $\omega_{l}, \Omega_{l} \in \mathcal{A}$ for $1 \leq l \leq k-2$. Thus, by Corollary 5 , $\Omega_{l}=d Q_{l}+q_{l} d H$ with $q_{l} \in \mathcal{S}$ for all $1 \leq l \leq k-2$, and by using Lemma 4 we conclude that $\bar{\Omega}_{k-1}:=$ $\sum_{i+j=l} q_{i} \omega_{j} \in \mathcal{A}$. Hence, since $\Omega_{k-1}=\omega_{k-1}+\bar{\Omega}_{k-1}$, Lemma 4 implies that $\omega_{k-1} \in \mathcal{A}$ if and only if $\Omega_{k-1} \in \mathcal{A}$.

(b) By Corollary $5, \Omega_{l}=d Q_{l}+q_{l} d H$ with $q_{l} \in \mathcal{S}$ for all $1 \leq l \leq k-1$, and $L_{1}(c) \equiv \cdots \equiv L_{k-1}(c) \equiv 0$. In addition, by the statement $(a), \omega_{l} \in \mathcal{A}$ for $1 \leq l \leq k-1$. Thus, $\bar{\Omega}_{k}:=\sum_{i+j=k} q_{i} \omega_{j} \in \mathcal{A}$ because of Lemma 4 , which implies that $\int_{\gamma_{c}} \bar{\Omega}_{k} \equiv 0$ by Corollary 5. Finally, from Theorem 2 we have $L_{k}(c)=\int_{\gamma_{c}} \omega_{k}+\int_{\gamma_{c}} \bar{\Omega}_{k}$. Therefore $L_{k}(c)=\int_{\gamma_{c}} \omega_{k}$.

Before announce next lemma, we note that each polynomial $h(x)=\sum_{r=0}^{m-1} a_{r} x^{r}$ of degree $m-1$ can be written as

$$
h(x)=\hat{h}\left(x^{2}\right)+x \tilde{h}\left(x^{2}\right)
$$


where

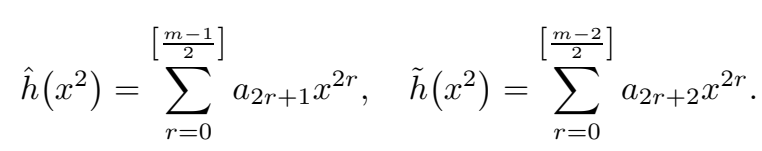

Lemma 8. Let $\omega=(g(x)+f(x) y) d x$, where $f(x)=\sum_{r=0}^{m-1} a_{r} x^{r}$ and $g(x)=\sum_{s=0}^{n} b_{s} x^{s}$.

(a) $\int_{\gamma_{c}} \omega=\int_{\gamma_{c}} \hat{f}\left(x^{2}\right) y d x$ and its value is

$$
-\pi c \sum_{r=0}^{\left[\frac{m-1}{2}\right]}\left(\begin{array}{c}
2(r+1) \\
r+1
\end{array}\right) \frac{a_{2 r+1}}{2^{r}(2 r+1)} c^{r}
$$

(b) If $\int_{\gamma_{c}} \omega \equiv 0$, then $\omega=d Q+(y \bar{q}) d H$ with $\bar{q} \in \mathbb{R}\left[x^{2}, H\right]$ of degree $\operatorname{deg}_{2} \bar{q}=[(m-2) / 2]$, and $\int_{\gamma_{c}}(y \bar{q}) \omega=$ $\int_{\gamma_{c}} \bar{q} \hat{g}\left(x^{2}\right) y d x$ whose value is

$$
-\pi c \sum_{s=0}^{\left[\frac{n}{2}\right]} \sum_{r=0}^{\left[\frac{m-2}{2}\right]}\left(\begin{array}{c}
2(s+r+1) \\
s+r+1
\end{array}\right) \frac{\left(b_{2 s}\right)\left(a_{2 r+2}\right)}{2^{s+r}(2 s+1)} c^{s+r} .
$$

(c) $\int_{\gamma_{c}}(y \bar{q}) \omega \equiv 0$ if and only if $\bar{q} \equiv 0$ or $\hat{g}\left(x^{2}\right) \equiv 0$.

Proof. (a) By statements $(a)$ and (b) of Lemma 3, $\int_{\gamma_{c}} \omega=\int_{\gamma_{c}} \hat{f}\left(x^{2}\right) y d x$, and the value of this integral follows from Corollary 6.

(b) If $\int_{\gamma_{c}} \omega \equiv 0$, then $\hat{f}\left(x^{2}\right) \equiv 0$ by $(a)$. This implies that $\omega=g(x) d x+x \tilde{f}\left(x^{2}\right) y d x$ and by (7) we have

$$
\omega=d\left(\int g(x) d x\right)+\sum_{r=0}^{\left[\frac{m-2}{2}\right]} a_{2 r+2} x^{2 r+1} y d x .
$$

From Lemma 3.(b) we obtain $x^{2 r+1} y d x=d\left(y \bar{Q}_{r}\right)+\left(y \bar{q}_{r}\right) d H$ for some homogeneous polynomials $\bar{Q}_{r}, \bar{q}_{r} \in$ $\mathbb{R}\left[x^{2}, H\right]$ with $\operatorname{deg}_{2} \bar{Q}_{r}=r+1$ and $\operatorname{deg}_{2} \bar{q}_{r}=r$, respectively. Hence

$$
\omega=d Q+(y \bar{q}) d H
$$

with

$$
Q=\int g(x) d x+y \sum_{r=0}^{\left[\frac{m-2}{2}\right]} a_{2 r+2} \bar{Q}_{r} \quad \text { and } \quad \bar{q}=\sum_{r=0}^{\left[\frac{m-2}{2}\right]} a_{2 r+2} \bar{q}_{r} .
$$

Thus $\bar{q} \in \mathbb{R}\left[x^{2}, H\right]$ is homogeneous and $\operatorname{deg}_{2} \bar{q}=\left[\frac{m-2}{2}\right]$. Moreover, a simple computation shows that

$$
\bar{q}_{r}=2 \sum_{i=0}^{r}\left(\begin{array}{c}
r+1 \\
i
\end{array}\right)\left(\frac{r+1-i}{2 i+1}\right)(2 H)^{r-i}\left(x^{2}-2 H\right)^{i} .
$$


As $(y \bar{q}) \omega=\bar{q} \hat{g}\left(x^{2}\right) y d x+\bar{q} \tilde{g}\left(x^{2}\right) x y d x+\bar{q} \tilde{f}\left(x^{2}\right) x y^{2} d x$ and $\bar{q} \tilde{f}\left(x^{2}\right) x y^{2} d x=\bar{q} \tilde{f}\left(x^{2}\right) x\left(2 H-x^{2}\right) d x$, it follows that $(y \bar{q}) \omega=\bar{q} \hat{g}\left(x^{2}\right) y d x+d Q_{2}+q_{2} d H$ because of statements $(a)$ and $(b)$ of Lemma 3 . Hence we obtain $\int_{\gamma_{c}}(y \bar{q}) \omega=\int_{\gamma_{c}} \bar{q} \hat{g}\left(x^{2}\right) y d x$. That is,

$$
\int_{\gamma_{c}}(y \bar{q}) \omega=\int_{\gamma_{c}}\left(\sum_{r=0}^{\left[\frac{m}{2}\right]-1} a_{2 r+2} \bar{q}_{r}\right)\left(\sum_{s=0}^{\left[\frac{n}{2}\right]} b_{2 s} x^{2 s}\right) y d x .
$$

By using expression (8) of $\bar{q}_{r}$, a straightforward computation, and Lemma 3(c) we obtain the formula given in the statement. Finally, statement $(c)$ follows from the formula given in statement $(b)$.

Remark 1. System $\left(1_{\varepsilon}\right)$ with $\mu=\nu=1, F_{1}(x)=-x^{2}$, and $g_{1}(x)=1-x^{2}$ does not satisfy the hypothesis in definition of $\mathcal{G} \mathcal{L} 1$ because $g_{1}(x)$ is not an odd function. Here $m=n=2$ and from Theorem 1.(a) it follows that $\tilde{\mathcal{H}}_{1}^{1}(2,2)=0$; however, for $\varepsilon$ small enough, this system has one medium amplitude limit cycle. Indeed, we need only to prove that the first non-vanishing coefficient of the displacement function (2), associated to this system, has a simple positive zero. For that we write system in the form $\left(3_{\varepsilon}\right)$ as $d H-\varepsilon \omega=0$ with $\omega=\left(1-x^{2}-2 x y\right) d x$. By Lemma 8. $(a), L_{1}(c) \equiv 0$, and by Theorem 2 and Lemma 8.(b), $L_{2}(c)=-\pi c(4-2 c)$.

Now, system $\left(1_{\varepsilon}\right)$ with $\mu=\nu=2, F_{1}(x)=-3 x^{2}, F_{2}(x)=-2 x^{3}, g_{1}(x)=x^{2}+x^{3}$, and $g_{2}(x)=$ $\left(-5+25 x^{2}\right) / 6$ does not satisfy the hypothesis in definition of $\mathcal{G} \mathcal{L} 2$ because $F_{2}(x)$ is not an even function. In this case $m=n=3$ and by Theorem 1.(b), $\overline{\mathcal{H}}_{2}^{2}(3,3)=1$; however, for $\varepsilon$ small enough, the resulting system has two medium amplitude limit cycles. Indeed, following previous ideas, and using Theorem 2 and Lemma 8 it is easy to see that $L_{1}(c) \equiv 0, L_{2}(c) \equiv 0$, and $L_{3}(c)=-\pi c(c-1)(c-2)$.

\section{Proof of the Theorem 1}

We can assume, after a linear change of variables if necessary, that $F_{i}(0)=0$ for all $1 \leq i \leq \mu$. Suppose that $F_{i}(x)=\sum_{r=1}^{m}\left(a_{i(r-1)} / r\right) x^{r}$ and $g_{i}(x)=\sum_{s=0}^{n} b_{i s} x^{s}$. Thus, $f_{i}(x)=F_{i}^{\prime}(x)=\sum_{r=0}^{m-1} a_{i r} x^{r}$ and $g_{i}(x)$ can be written as $f_{i}(x)=\hat{f}_{i}\left(x^{2}\right)+x \tilde{f}_{i}\left(x^{2}\right)$ and $g_{i}(x)=\hat{g}_{i}\left(x^{2}\right)+x \tilde{g}_{i}\left(x^{2}\right)$, respectively, according to (6).

Proof of Theorem 1. (a) By hypothesis, $g_{i}(x)$ is odd for $1 \leq i \leq \nu$, which means that $g_{i}(x)=x \tilde{g}_{i}\left(x^{2}\right)$ for $1 \leq i \leq \nu$. Let $L_{k}(c)$ be the first non-vanishing Poincaré-Pontryagin-Melnikov function in (2). For proving the statement we will prove first that $L_{k}(c)$ has at most $[(m-1) / 2]$ positive zeros, and then that for each $s$ with $0 \leq s \leq[(m-1) / 2]$ we can choose systems in $\mathcal{G} \mathcal{L} 1$ in such a way that $L_{k}(c)$ has exactly $s$ simple positive zeros.

If $k=1$, then the assertion is true. Indeed, we have

$$
L_{1}(c)=\int_{\gamma_{c}} x \tilde{g}_{i}\left(x^{2}\right) d x+\hat{f}_{1}\left(x^{2}\right) y d x+\tilde{f}_{1}\left(x^{2}\right) x y d x .
$$

Thus, as $\int_{\gamma_{c}} x \tilde{g}_{i}\left(x^{2}\right) d x \equiv 0$ and $\int_{\gamma_{c}} \tilde{f}_{1}\left(x^{2}\right) x y d x \equiv 0$ by Corollary 5, we obtain $L_{1}(c)=\int_{\gamma_{c}} \hat{f}_{1}\left(x^{2}\right) y d x$. From (7) we know that $\operatorname{deg}_{2} \hat{f}_{1}\left(x^{2}\right)=[(m-1) / 2]$, which implies that $L_{1}(c)$ has at most $[(m-1) / 2]$ positive zeros because of Corollary 6 . In addition, since $\hat{f}_{1}\left(x^{2}\right)$ has $[(m-1) / 2]+1$ independent coefficients, for each $s$ with $0 \leq s \leq[(m-1) / 2]$ we can choose suitable coefficients of $\hat{f}_{1}\left(x^{2}\right)$ in such a way that $L_{k}(c)$ has exactly $s$ simple positive zeros. Therefore, by applying the Poincaré-Pontryagin-Andronov criterion it follows that 
the corresponding system $\left(1_{\varepsilon}\right)$, which belongs to $\mathcal{G L} 1$, has exactly $s$ hyperbolic limit cycles. In particular we have proved that $\tilde{\mathcal{H}}_{\nu}^{\mu}(m, n)=[(m-1) / 2]$.

Suppose then that $k \geq 2$. If we prove that $\Omega_{l} \in \mathcal{A}$ for $1 \leq l \leq k-1$, then $L_{k}(c)=\int_{\gamma_{c}} \omega_{k}$ by Lemma 7 , and by applying the same idea as in previous paragraph the assertion follows. Therefore, it remains to show that $\Omega_{l} \in \mathcal{A}$ for $1 \leq l \leq k-1$.

We proceed by induction on $k$. If $k=2$, then $L_{1}(c) \equiv 0$, which implies that

$$
\Omega_{1}=\left(x \tilde{g}_{1}\left(x^{2}\right)+x y \tilde{f}_{1}\left(x^{2}\right)\right) d x \in \mathcal{A} .
$$

Hence the assertion is true for $k=2$.

We now assume that the assertion is true for $k-2$, and we will prove it for $k-1$. By induction hypothesis, $\Omega_{i} \in \mathcal{A}$ for $1 \leq i \leq k-2$, which implies that $\Omega_{i}=d Q_{i}+q_{i} d H$ with $q_{i} \in \mathcal{S}$ for $1 \leq i \leq k-2$ by Corollary 5 . Furthermore, by Lemma $7, \omega_{j} \in \mathcal{A}$ for $1 \leq j \leq k-2$. Hence $\bar{\Omega}_{k-1}:=\sum_{i+j=k-1} q_{i} \omega_{j}($ with $1 \leq i, j \leq k-2)$ is an element of $\mathcal{A}$ because of Lemma 4 . Since $\Omega_{k-1}=\omega_{k-1}+\bar{\Omega}_{k-1}$,

$$
L_{k-1}(c)=\int_{\gamma_{c}} \Omega_{k-1}=\int_{\gamma_{c}} \omega_{k-1}=\int_{\gamma_{c}} \hat{f}_{k-1}\left(x^{2}\right) y d x
$$

which vanishes identically. Therefore, $\omega_{k-1}=\left(x \tilde{g}_{k-1}\left(x^{2}\right)+x y \tilde{f}_{k-1}\left(x^{2}\right)\right) d x \in \mathcal{A}$. Thus $\Omega_{k-1} \in \mathcal{A}$.

(b) Firstly we will show two properties concerning $\omega_{i}$ and $\int_{\gamma_{c}} \omega_{i}$ which we will use along the proof of the statement. Then, we will split the proof into two cases: $m$ odd and $m$ even.

For $1 \leq i<\mu_{0}$ the 1 -form $\omega_{i}$ has the form $g_{i}(x) d x$ that is exact: $\omega_{i}=d Q_{i}+q_{i} d H$ with $q_{i} \equiv 0$. Hence, from (4) it follows that $\Omega_{i}=\omega_{i}$ for $1 \leq i \leq \mu_{0}$. Thus, $L_{i}(c)=\int_{\gamma_{c}} \Omega_{i} \equiv 0$ for $1 \leq i<\mu_{0}$ and $L_{\mu_{0}}(c)=$ $\int_{\gamma_{c}} \Omega_{\mu_{0}}=\int_{\gamma_{c}} \omega_{\mu_{0}}$. On the other hand, since $F_{i}(x)$ is even for $\mu_{0}<i \leq \mu, f_{i}(x)=x \tilde{f}_{i}\left(x^{2}\right)$ for $\mu_{0}<i \leq \mu$. Thus, for $i>\mu_{0}$ we have $\omega_{i}=d\left(\int g_{i}(x) d x\right)+x \tilde{f}_{i}\left(x^{2}\right) y d x$. Moreover, $x^{2 r+1} y d x=d\left(y \bar{Q}_{0 r}\right)+\left(y \bar{q}_{0 r}\right) d H$ because of Lemma 3.(b). Hence,

$$
\omega_{i}=d\left(\bar{Q}_{i}\right)+\left(y \bar{q}_{i}\right) d H
$$

of course $\bar{q}_{i} \equiv 0$ for $i>\mu$. Therefore we obtain

$$
\int_{\gamma_{c}} \omega_{i} \equiv 0 \quad \text { for } i>\mu_{0}
$$

Case $m$ odd. In this case $\operatorname{deg} F_{\mu_{0}}(x)=m$ because $F_{i}(x)$ is an even polynomial for $\mu_{0}<i \leq \mu$. Since $F_{\mu_{0}}^{\prime}(x)=f_{\mu_{0}}(x)=\hat{f}_{\mu_{0}}\left(x^{2}\right)+x \tilde{f}_{\mu_{0}}\left(x^{2}\right)$ has an even degree, $\hat{f}_{\mu_{0}}\left(x^{2}\right) \not \equiv 0$. Hence, from Lemma 8.(a) it follows that $L_{\mu_{0}}(c)=\int_{\gamma_{c}} \omega_{\mu_{0}}=\int_{\gamma_{c}} \hat{f}_{\mu_{0}}\left(x^{2}\right) y d x \not \equiv 0$, and it has at most $[(m-1) / 2]$ positive zeros, counting multiplicities; moreover, we can choose suitable coefficients of $F_{\mu_{0}}(x)$ in such a way that $L_{\mu_{0}}(c)$ has exactly $[(m-1) / 2]$ simple positive zeros. Therefore by the Poincaré-Pontryagin-Andronov criterion, $\overline{\mathcal{H}}_{\nu}^{\mu}(m, n)=[(m-1) / 2]$.

Case $m$ even. Let $L_{k}(c)$ be the first non-vanishing Poincaré-Pontryagin-Melnikov function of (2). If $k=\mu_{0}$, then $L_{\mu_{0}}(c)=\int_{\gamma_{c}} \omega_{\mu_{0}}$ has at most $[(m-1) / 2]$ positive zeros, counting multiplicities, because of Lemma 8. $(a)$. Since $m$ is even, $[(m-1) / 2] \leq[m / 2]+[n / 2]-1$. Hence $L_{\mu_{0}}(c)$ has at most $[m / 2]+[n / 2]-1$ positive zeros, counting multiplicities.

We claim that if $k \geq \mu_{0}+1$, then $\omega_{1}, \ldots, \omega_{k-1-\mu_{0}} \in \mathcal{A}, \Omega_{i}=d Q_{i}+q_{i} d H$ with $q_{i} \in \mathcal{S}$ for $\mu_{0} \leq i \leq$ $k-1$, and $L_{k}(c)=\int_{\gamma_{c}}\left(y \bar{q}_{\mu_{0}}\right) \omega_{k-\mu_{0}}=\int_{\gamma_{c}} \bar{q}_{\mu_{0}} \hat{g}_{k-\mu_{0}}\left(x^{2}\right) y d x$. By assuming that this assertion is true and 
by applying Lemma 8.(b) we conclude that $L_{k}(c)$ has at most $[m / 2]+[n / 2]-1$ positive zeros, counting multiplicities; moreover, for each $s$ with $0 \leq s \leq[m / 2]+[n / 2]-1$ we can choose suitable coefficients of $\bar{q}_{\mu_{0}}$ and $\hat{g}_{k-\mu_{0}}\left(x^{2}\right)$ in such a way that $L_{k}(c)$ has exactly $s$ simple positive zeros. Thus, by the PoincaréPontryagin-Andronov criterion the corresponding system $\left(1_{\varepsilon}\right)$ has exactly $s$ hyperbolic limit cycles; in particular we have $\overline{\mathcal{H}}_{\nu}^{\mu}(m, n)=[m / 2]+[n / 2]-1$. Therefore, to finish the proof of statement $(b)$ we need only to confirm our assertion, which we prove next by proceeding by induction on $k$.

If $k=\mu_{0}+1$, then we will prove that $\Omega_{\mu_{0}}=d Q_{\mu_{0}}+q_{\mu_{0}} d H$ with $q_{\mu_{0}} \in \mathcal{S}$, and that $L_{\mu_{0}+1}(c)=$ $\int_{\gamma_{c}} \bar{q}_{\mu_{0}} \hat{g}_{1}\left(x^{2}\right) y d x$. We know that $\Omega_{\mu_{0}}=\omega_{\mu_{0}}$ and since $k=\mu_{0}+1, L_{\mu_{0}}(c)=\int_{\gamma_{c}} \Omega_{\mu_{0}} \equiv 0$. Thus, from Lemma 8.(b) it follows that $\Omega_{\mu_{0}}=\omega_{\mu_{0}}=d Q_{\mu_{0}}+q_{\mu_{0}} d H$, where $q_{\mu_{0}}=y \bar{q}_{\mu_{0}} \not \equiv 0 \in \mathcal{S}$. On the other hand, by Theorem 2, $L_{\mu_{0}+1}(c)=\int_{\gamma_{c}} \Omega_{\mu_{0}+1}$, where $\Omega_{\mu_{0}+1}=\omega_{\mu_{0}+1}+\sum_{i+j=\mu_{0}+1} q_{i} \omega_{j}$. Since $q_{i} \equiv 0$ for $1 \leq i<\mu_{0}$, $\Omega_{\mu_{0}+1}=\omega_{\mu_{0}+1}+q_{\mu_{0}} \omega_{1}$. Hence, by (10) we obtain $L_{\mu_{0}+1}(c)=\int_{\gamma_{c}}\left(y \bar{q}_{\mu_{0}}\right) \omega_{1}=\int_{\gamma_{c}} \bar{q}_{\mu_{0}} \hat{g}_{1}\left(x^{2}\right) y d x$.

If $k=\mu_{0}+2$, then $\Omega_{\mu_{0}}=\omega_{\mu_{0}}=d Q_{\mu_{0}}+q_{\mu_{0}} d H$, where $q_{\mu_{0}}=y \bar{q}_{\mu_{0}} \not \equiv 0 \in \mathcal{S}$ and $L_{\mu_{0}+1}(c) \equiv 0$. Since $\bar{q}_{\mu_{0}} \not \equiv 0, \hat{g}_{1}\left(x^{2}\right) \equiv 0$ by Lemma 8.(c). Thus, $\Omega_{1}=\omega_{1} \in \mathcal{A}$, and Corollary 5 implies that $\Omega_{1}=d Q_{1}+q_{1} d H$ with $q_{1} \in \mathcal{S}$. Moreover, from (9) $\omega_{\mu_{0}+1}=d\left(\bar{Q}_{\mu_{0}+1}\right)+\left(y \bar{q}_{\mu_{0}+1}\right) d H$, whence

$$
\Omega_{\mu_{0}+1}=\omega_{\mu_{0}+1}+q_{\mu_{0}} \omega_{1}=d Q_{\mu_{0}+1}+q_{\mu_{0}+1} d H
$$

with $q_{\mu_{0}+1} \in \mathcal{S}$ because of Corollary 5 .

On the other hand, from Theorem 2 we have

$$
L_{\mu_{0}+2}(c)=\int_{\gamma_{c}} \omega_{\mu_{0}+2}+\sum_{i+j=\mu_{0}+2} \int_{\gamma_{c}} q_{i} \omega_{j} .
$$

As $\omega_{1} \in \mathcal{A}$ and $q_{\mu_{0}+1} \in \mathcal{S}$, then $q_{\mu_{0}+1} \omega_{1} \in \mathcal{A}$ following Lemma 4 and $\int_{\gamma_{c}} q_{\mu_{0}+1} \omega_{1} \equiv 0$ by Corollary 5 . In addition, we know that $q_{i} \equiv 0$ for $1 \leq i<\mu_{0}$ and $\int_{\gamma_{c}} \omega_{\mu_{0}+2} \equiv 0$ by $(10)$. Hence $L_{\mu_{0}+2}(c)=\int_{\gamma_{c}}\left(y \bar{q}_{\mu_{0}}\right) \omega_{2}=$ $\int_{\gamma_{c}} \bar{q}_{\mu_{0}} \hat{g}_{2}\left(x^{2}\right) y d x$.

We now assume that the assertion holds for $k-1$, and we will prove it for $k$. By Theorem $2, L_{k}(c)=$ $\int_{\gamma_{c}} \Omega_{k}$, where

$$
\Omega_{k}=\omega_{k}+q_{1} \omega_{k-1}+\cdots+q_{\mu_{0}-1} \omega_{k+1-\mu_{0}}+q_{\mu_{0}} \omega_{k-\mu_{0}}+q_{\mu_{0}+1} \omega_{k-1-\mu_{0}}+\cdots+q_{k-2} \omega_{2}+q_{k-1} \omega_{1} .
$$

Since $q_{i} \equiv 0$ for $1 \leq i<\mu_{0}, \Omega_{k}=\omega_{k}+q_{\mu_{0}} \omega_{k-\mu_{0}}+q_{\mu_{0}+1} \omega_{k-1-\mu_{0}}+\cdots+q_{k-2} \omega_{2}+q_{k-1} \omega_{1}$.

On the other hand, from the induction hypothesis it follows that $\omega_{1}, \ldots, \omega_{k-2-\mu_{0}} \in \mathcal{A}, \Omega_{i}=d Q_{i}+q_{i} d H$ with $q_{i} \in \mathcal{S}$ for $\mu_{0} \leq i \leq k-2$, and $L_{k-1}(c)=\int_{\gamma_{c}} \bar{q}_{\mu_{0}} \hat{g}_{k-1-\mu_{0}}\left(x^{2}\right) y d x$. Since $L_{k-1}(c) \equiv 0, \hat{g}_{k-1-\mu_{0}}\left(x^{2}\right) \equiv 0$ because of Lemma 8.(c), which implies that $\omega_{k-1-\mu_{0}} \in \mathcal{A}$. Therefore, $q_{\mu_{0}} \omega_{k-1-\mu_{0}}+\cdots+q_{k-3} \omega_{2}+q_{k-2} \omega_{1} \in \mathcal{A}$ by Lemma 4 . Moreover, from $(10) \omega_{k-1}=d\left(\bar{Q}_{k-1}\right)+\left(y \bar{q}_{k-1}\right) d H$, and by applying Corollary 5 we obtain

$$
\Omega_{k-1}=d Q_{k-1}+q_{k-1} d H, \quad \text { with } q_{k-1} \in \mathcal{S} .
$$

Hence $q_{\mu_{0}+1} \omega_{k-1-\mu_{0}}+\cdots+q_{k-2} \omega_{2}+q_{k-1} \omega_{1} \in \mathcal{A}$ by Lemma 4 . In addition, $\omega_{k}=d\left(\bar{Q}_{k}\right)+\left(y \bar{q}_{k}\right) d H$ by (10). Thus, we obtain $L_{k}(c)=\int_{\gamma_{c}} q_{\mu_{0}} \omega_{k-\mu_{0}}=\int_{\gamma_{c}} \bar{q}_{\mu_{0}} \hat{g}_{k-\mu_{0}}\left(x^{2}\right) y d x$.

\section{Acknowledgments}

Part of the results of this work come from the author's postdoctoral stay at the Departament de Matemàtiques of the Universitat Autònoma de Barcelona. The author would like to thank the Centre de Recerca Matemàtica for their support and hospitality during the period in which this paper was completed. 


\section{References}

[1] Caubergh, M. \& Françoise, J.P. [2004] "Generalized Liénard equations, cyclicity and Hopf-Takens bifurcations," Qual. Theory Dyn. Syst. 5, pp. 195-222.

[2] Christopher, C. \& Li, C. [2007] Limit Cycles of Differential Equations, Advanced Courses in Mathematics. CRM Barcelona. Birkhäuser Verlag, Basel.

[3] Françoise, J.P. [1996] "Successive derivatives of a first return map, application to the study of quadratic vector fields," Ergodic Theory Dynam. Systems 16, pp. 87-96.

[4] García-Saldaña, J.D., Gasull, A., \& Giacomini, H. [2012] "Bifurcation values for a family of planar vector fields of degree five. To appear in Discrete Contin. Dyn. Syst. 35.

[5] Gasull, A. [1989] "Differential equations that can be transformed into equations of Liénard type," in Actas del XVII Colloquio Brasileiro de Matemática.

[6] Gasull A. \& Giacomini H. [2010] "Upper bounds for the number of limit cycles of some planar polynomial differential systems," Discrete Contin. Dyn. Syst. 27, pp. 217-229.

[7] Gasull, A. \& Torregrosa, J. [2001] "A relation between small amplitude and big limit cycles," Rocky Mountain J. Math. 31, pp. 1277-1303.

[8] Gavrilov, L. [1998] "Petrov modules and zeros of Abelian integrals," Bull. Sci. Math. 122, pp. 571-584.

[9] Gavrilov, L. [2008] "Cyclicity of period annuli and principalization of Bautin ideals," Ergodic Theory Dynam. Systems 28, pp. 1497-1507.

[10] Glade, N., Forest, L. \& Demongeot, J. [2007] "Liénard systems and potential-Hamiltonian decomposition. III. Applications," C. R. Math. Acad. Sci. Paris 344, pp. 253-258.

[11] Iliev, I. D. [1999] "The number of limit cycles due to polynomial perturbations of the harmonic oscillator," Math. Proc. Cambridge Philos. Soc. 127, pp. 317-322.

[12] Ilyashenko, Y. [1969] "The appearance of limit cycles under a perturbation of the equation $\frac{d w}{d z}=-\frac{R_{z}}{R_{w}}$, where $R(z, w)$ is a polynomial," Mat. Sb. (N.S.) 78, pp. 360373 (in Russian).

[13] Ilyashenko, Y. [2002] "Centennial history of Hilbert's 16th problem," Bull. Amer. Math. Soc. (N.S.) 39, pp. 301-354.

[14] Ilyashenko, Y. \& Yakovenko, S. [2008] Lectures on Analytic Differential Equations, Graduate Studies in Mathematics, 86. American Mathematical Society, Providence, RI.

[15] Leonov, G. [2008] "Hilbert's 16th problem for quadratic systems. New methods based on a transformation to the Liénard equation," Int. J. Bifurcation and Chaos 18, pp. 877-884.

[16] Li, J. [2003] "Hilbert's 16th problem and bifurcations of planar polynomial vector fields," Int. J. Bifurcation and Chaos 13, pp. 47-106.

[17] Lins, A., de Melo, W. \& Pugh, C. C. [1977] "On Liénard's equation," Lecture Notes in Math., 597, Springer, Berlin, pp. 335-357. 
[18] Llibre, J. [2009] "A survey on the limit cycles of the generalization polynomial Liénard differential equations," Mathematical models in engineering, biology and medicine, AIP Conf. Proc., 1124, Amer. Inst. Phys., Melville, NY. pp 224-233.

[19] Llibre, J., Mereu, A. C. \& Teixeira, M. A. [2010] "Limit cycles of the generalized polynomial Liénard differential equations," Math. Proc. Cambridge Philos. Soc. 148, pp. 363-383.

[20] Moreira, H. N. [1992] "Liénard-type equations and the epidemiology of malaria," Ecological modelling 60, pp. 139-150.

[21] Perko, L. M. [2001] Differential Equations and Dynamical Systems, 3rd ed., Springer-Verlag, New York.

[22] Rebollo-Perdomo, S. [2009] "The infinitesimal Hilbert's 16th problem in the real and complex planes," Qual. Theory Dyn. Syst. 7, pp. 467-500.

[23] Yakovenko, S. [1995] "A geometric proof of the Bautin theorem," Concerning the Hilbert 16th problem. Amer. Math. Soc. Transl. Ser. 2, 165, pp. 203-219. 